\title{
RESEARCH
}

Open Access

\section{Effectiveness of HIIT compared to moderate continuous training in improving vascular parameters in inactive adults}

Robinson Ramírez-Vélez ${ }^{1 *}$ (D), Paula Andrea Hernández-Quiñones², Alejandra Tordecilla-Sanders ${ }^{1}$, Cristian Álvarez ${ }^{3,4}$, Rodrigo Ramírez-Campillo ${ }^{4}$, Mikel Izquierdo ${ }^{5}$, Jorge Enrique Correa-Bautista ${ }^{1}$, Antonio Garcia-Hermoso ${ }^{6}$ and Ronald G. Garcia ${ }^{7,8,9}$

\begin{abstract}
Background: Strong evidence shows that physical inactivity increases the risk of many adverse health conditions, including major non-communicable diseases, such as cardiovascular disease (CVD), metabolic syndrome, and breast and colon cancers, and shortens life expectancy. We aimed to determine the effects of moderate (MCT)- versus high-intensity interval training (HIT) on vascular function parameters in physically inactive adults. We hypothesized that individualized HIT prescription would improve the vascular function parameters more than the MCT in a greater proportion of individuals.
\end{abstract}

Methods: Twenty-one inactive adults were randomly allocated to receive either MCT group $(60-75 \%$ of their heart rate reserve, [HRR] or HIT group (4 min at 85-95\% of peak HRR), 3 days a week for 12 weeks. Vascular function (brachial artery flow-mediated dilation, FMD [\%], normalized brachial artery flow-mediated dilation, FMDn [\%], aortic pulse wave velocity, PWV $\left[\mathrm{m} \cdot \mathrm{s}^{-1}\right]$, Alx, augmentation index: aortic and brachial [\%]), were measured at baseline and over 12 weeks of training. In order for a participant to be considered a responder to improvements in vascular function parameters (FMDn and PWV), the typical error was calculated in a favorable direction.

Results: FMD changed by $-1.0 \%$ (SE 2.1, $d=0.388$ ) in the MCT group, and $+1.8 \%$ (SE 1.8, $d=0.699$ ) in the HIT group (no significant difference between groups: $2.9 \%$ [95\% Cl, -3.0 to 8.8]. PW changed by $+0.1 \mathrm{~m} \cdot \mathrm{s}^{-1}(\mathrm{SE} 0.2, d=0.087$ ) in the MCT group but decreased by $-0.4 \mathrm{~m} \cdot \mathrm{s}^{-1}$ in the HIT group (SE $0.2, d=0.497$ ), with significant difference between groups: $-0.4[95 \% \mathrm{Cl},-0.2$ to -0.7$]$. There was not a significant difference in the prevalence of no-responder for FMD (\%) between the MCT and HIT groups (66\% versus $36 \%, P=0.157)$. Regarding PW $\left(\mathrm{m} \cdot \mathrm{s}^{-1}\right)$, an analysis showed that the prevalence of no-responder was 77\% (7 cases) in the MCT group and $45 \%$ (5 cases) in the HIT group ( $P=0.114$ ).

Conclusions: Under the conditions of the present study, both groups experienced changed in vascular function parameters. Compared to MCT group, HIT is more efficacious for improving FMD and decreasing PWW, in physically inactive adults.

Trial registration: ClinicalTrials.gov NCT02738385 registered on 23 March 2016.

Keywords: Aerobic exercise, Arterial stiffness, Cardiovascular disease prevention, Endothelial dysfunction, Sedentarism

\footnotetext{
*Correspondence: robin640@hotmail.com; robinson.ramirez@urosario.edu.co

${ }^{1}$ Centro de Estudios para la Medición de la Actividad Física "CEMA". Escuela

de Medicina y Ciencias de la Salud, Universidad del Rosario, Bogotá, DC,

Colombia

Full list of author information is available at the end of the article
}

(C) The Author(s). 2019 Open Access This article is distributed under the terms of the Creative Commons Attribution 4.0 International License (http://creativecommons.org/licenses/by/4.0/), which permits unrestricted use, distribution, and reproduction in any medium, provided you give appropriate credit to the original author(s) and the source, provide a link to the Creative Commons license, and indicate if changes were made. The Creative Commons Public Domain Dedication waiver (http://creativecommons.org/publicdomain/zero/1.0/) applies to the data made available in this article, unless otherwise stated. 


\section{Background}

Strong evidence shows that physical inactivity $(<150 \mathrm{~min} /$ wk. of moderate-intensity activity or $<75 \mathrm{~min} / \mathrm{wk}$. of high -intensity activity) increases the risk of many adverse health conditions, including major non-communicable diseases, such as cardiovascular disease (CVD), metabolic syndrome, and breast and colon cancers, and shortens life expectancy $[1,2]$. Physical inactivity has a deleterious effect that is comparable to smoking and obesity and is now recognized as the fourth leading risk factor for global mortality, accounting for $6 \%$ of all deaths [2].

Growing evidence suggests that exercise training improves vascular structure and nitric oxide bioavailability and reduces CVD risk factors; improvements in endothelial function may explain a large proportion of the risk reduction [3]. A number of factors appear to influence the acute effects of exercise on endothelial function, including sex, exercise intensity and duration, and the timing of post-exercise vascular function measurements [3]. Training protocols involving traditional moderate continuous training (MCT) and high-intensity training (HIT) can improve endothelial function $[4,5]$ a response largely mediated by acute elevations in blood flow and laminar shear stress during individual exercise bouts $[6,7]$. In line with this, a growing body of evidence has demonstrated comparable or superior improvements in cardiovascular function using low-volume HIT compared to MCT [5].

Additionally, three sessions of 4 min of HIT per week (12 min/week) was sufficient to improve aortic reservoir pressure (an independent predictor of CVD), and thus may be a time-efficient exercise modality for reducing cardiovascular risk in individuals with metabolic syndrome $[5,8]$. Furthermore, it was suggested that the ability of HIT to restore vascular homeostasis through the enhancement of shear stress-induced nitric oxide bioavailability may be another important mechanism that explains the protective role of exercise against non-communicable disease development [9]. Interestingly, despite this evidence, few randomized trials have directly evaluated the effects of sustained MCT or HIT on the cardiometabolic health of inactive adults $[4,9,10]$.

There exists an inter-individual variability in vascular function, such that under the same stimulus, some subjects may achieve benefits, and are considered responders (Rs), whereas others may exhibit a worsened response or remain unchanged, and are considered non-responders (NRs) [11, 12]. Both genetic and environmental factors have been described to explain this previously reported phenomenon $[13,14]$. However, all of these studies are primarily endurance or resistance training-based $[9,15]$ and most have not explored other exercise modalities such as HIT [16].

In Latin-American populations, information about optimal exercise timing for improving vascular function parameters is scarce. There is no consensus regarding optimal exercise timing for improving vascular function parameters. Additionally, determining the prevalence of "NRs" after an exercise program is relevant to optimize and predict responses in different populations (e.g., athletes or individuals with cardiometabolic risk factors).

The purpose of this secondary randomized clinical trial analysis was to compare the effects of MCT versus HIT on vascular function in physically inactive adult Latin-Americans. We hypothesized that individualized HIT prescription would improve the vascular function parameters more than the MCT in a greater proportion of individuals. Identifying the training regimen that has the most beneficial effects on each parameter could potentially lead to enhanced precision in prescribing exercise training intensity to achieve optimal outcomes in this population [16].

\section{Methods \\ Sample and procedures}

Details of the study design and methods of the primary HIT-Heart Study trial have been described elsewhere (ClinicalTrials.gov ID: NCT02738385) [17, 18]. Informed consent was obtained from each participant. The protocol was based on the Helsinki Declaration Accord (World Medical Association for Human Subjects). Moreover, ethical approval was obtained from the University of Santo Tomás (ID 27-0500-2015). Vascular function and fitness parameters were assessed at baseline and over 12 weeks of training. Briefly, the HIT-Heart Study conducted in 2013-2015 tested the efficacy of MCT versus HIT in changings biomarkers of endothelial and cardiovascular health (see Additional file 1: Figure S1 for CONSORT diagram).

Participants $(n=21)$ were recruited at the University of Rosario (Bogota, Colombia) from February 2015 to May 2016. Inclusion criteria were individuals aged $18-45$ years who were inactive $\left(<150 \mathrm{~min} \cdot \mathrm{wk}^{-1}\right.$ of moderate-intensity activity or $75 \mathrm{~min} \cdot \mathrm{wk}^{-1}$ of vigorous-intensity activity by applied a short version of the self-reported Global Physical Activity Questionnaire) and had a body mass index (BMI) $\geq 18$ and $\leq 30 \mathrm{~kg} / \mathrm{m}^{2}$. We excluded participants if they had a history of cardiovascular disease and related morbidities, diabetes mellitus 1 or 2 , thyroid dysfunction, or cancer or if they were pregnant or smoked. All participants provided written informed consent before participating in the study. Participants were randomly assigned via a computer-generated, concealed, fixed block randomisation procedure to MCT $(n=10)$ or HIT $(n=11)$ groups. Data were obtained prior to randomisation by treating physiotherapists and physiologist, and then 12 weeks later by blinded assessors. Assessments were taken at baseline (Week 0) and 12 weeks after randomisation for all outcomes by experienced and blinded physiotherapists or exercise physiologist. 


\section{Interventions}

\section{Moderate-continuous training (MCT) group}

The MCT protocol involved walking on a treadmill with the deck inclined to reach the desired intensity. Each preparatory period started with an exercise dose of $6 \mathrm{kcal} \cdot \mathrm{kg}^{-1} \cdot$ week $^{-1}$, which was increased progressively by $2 \mathrm{kcal} \cdot \mathrm{kg}^{-1} \cdot$ week $^{-1}$ until week 4 and was then maintained at $12 \mathrm{kcal} \cdot \mathrm{kg}^{-1} \cdot$ week $^{-1}$ for weeks 5 to 12 . Exercise training sessions were designed to elicit a response in the acceptable moderate range, i.e., $60-75 \%$ of HRR and were adjusted according to ratings on the Borg scale [17, 18]. The rating of perceived exertion used was 12 to 15 -point single-item scale ranging from 6 to 20 (6 "No exertion" and 20 "Maximum exertion"). Sessions consisted of a warm-up walk (10 min), followed by an aerobic exercise session (30-35 min) and a final relaxation/cool-down period $(4 \mathrm{~min})$. Exercise was performed in three sessions per week. During the supervised intervention, HR was recorded using a HR monitor (Polar Pacer, USA) to ensure compliance with the exercise stimulus at the predetermined target $\mathrm{HR}$ zone (Fig. 1).

\section{High-intensity training (HIT) group}

The HIT protocol involved fast walking and running on a treadmill with the deck inclined to reach the desired intensity. We calculated training energy expenditures according to participants' age ranges associated with meeting the consensus public health recommendations from the Cardiometabolic HIT-RT Study [17, 18]. Each preparatory period started with an exercise dose of 6 $\mathrm{kcal} \cdot \mathrm{kg}^{-1} \cdot$ week $^{-1}$, which was increased progressively by 2 $\mathrm{kcal} \cdot \mathrm{kg}^{-1} \cdot$ week $^{-1}$ until week 4 and was then maintained at
$12 \mathrm{kcal} \cdot \mathrm{kg}^{-1} \cdot$ week $^{-1}$ for weeks 5 to 12 . The overall goal for the HIT group was to perform exercise sessions in $4 \times 4$ min intervals at $85-95 \%$ of HRR (with the target zone maintained for at least $2 \mathrm{~min}$ ), interspersed with a 4-min recovery period at $75-85 \%$ of HRR. The speed and inclination of the treadmill were continuously adjusted to ensure that participants trained at the correct intensity. During each exercise session, participants adhered to the $12-\mathrm{kcal} \cdot \mathrm{kg}^{-1} \cdot \mathrm{week}^{-1}$ energy expenditure format, which was equivalent to $300 \mathrm{kcal}$ of energy expended by the end of the training and cool-down $(4 \mathrm{~min})$ periods, with a total exercise time ranging from 38 to $42 \mathrm{~min}$. Exercise was performed in three sessions per week. During the supervised intervention, $\mathrm{HR}$ and Borg ratings were measured as described for the MCT group.

We selected 6 to $12 \mathrm{kcal} \cdot \mathrm{kg}^{-1} \cdot$ week $^{-1}$ per week because this dose of $\mathrm{kcal} \cdot \mathrm{kg}^{-1} \cdot$ week $^{-1}$ has produced changes in $\mathrm{VO}_{2}$ peak that placed approximately $70 \%$ of the initial sedentary population above the cut point for a low level of fitness [17, 18], as defined by both the American College of Sports Medicine (ACSM) [19] and the American Heart Association [20] guidelines for cardiovascular disease reduction.

The intensity to run/walk was related to a range of $85-95 \%$ (HIT) or $60-75 \%$ (MCT) of the maximum predicted heart rate according to the widely known equation (Karvonen), and the rest period was considered under a heart rate of $75-85 \%$ to HIT group of this marker. Thus, using the heart rate and oxygen consumption data obtained from the baseline fitness (cardiorespiratory uptake) test, the heart rate associated with an oxygen consumption of approximately

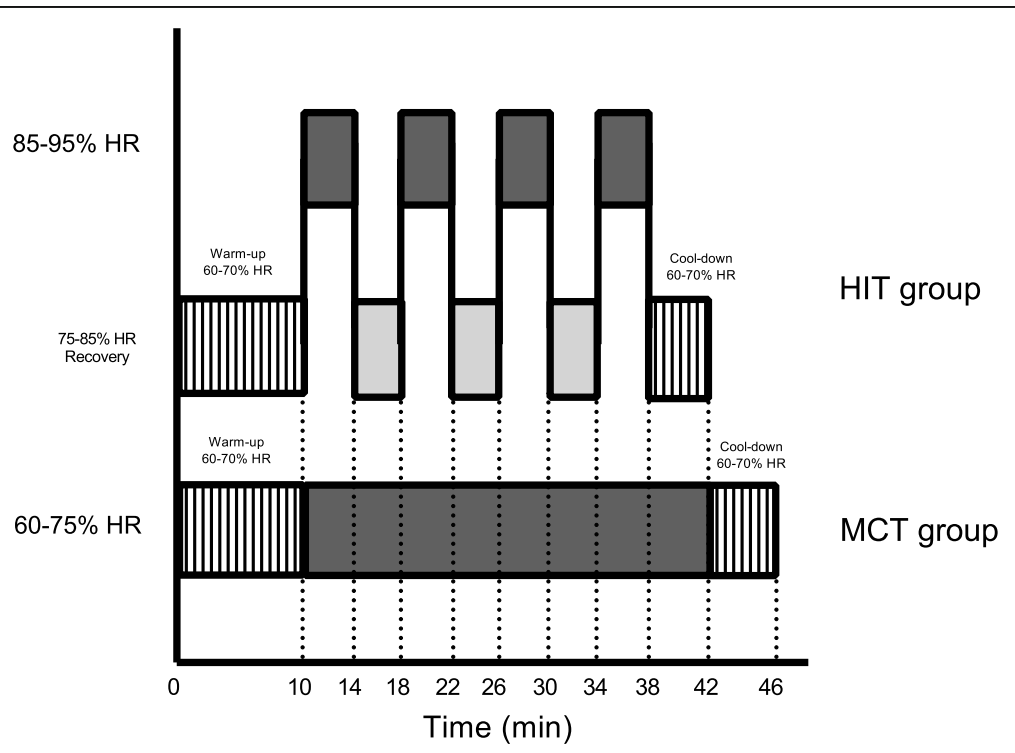

Fig. 1 Schematic representation of a $4 \times 4$ HIT session (35-38 min) or MCT session (38-43 min) 
60\% (MCT) and approximately 75-85\% (HIT) were prescribed for each participant [19].

\section{Endothelial function arterial and wall parameters measures}

The primary outcome measure was vascular function, as measured by flow mediated-dilation (FMD), aortic pulse wave velocity (PWV) and the augmentation index (Aix: aortic and brachial). FMD was measured as described in previous studies from our group [21] in the Colombian population using the protocol reported by Atkinson et al. [22]. A detailed description of the FMD technique can be found in a previous study ${ }^{21}$. FMD was expressed as $\%$ change $=[$ (maximum - baseline diameter $) /$ baseline diameter $\times 100$. Normalized brachial artery FMD (FMDn) was calculated according allometric relationship between Diameter (D) base and peak diameter (Dpeak), both measures in base-10 logarithm [22].

PWV was measured by analyzing the oscillometric pressure curves registered from the upper arm with arteriographic computer program (Arteriograph Software v.1.9.9.2; TensioMed, Budapest, Hungary). The algorithm measuring blood pressure in the arteriography device has been validated [23]. A detailed description of the PWV and AIx technique can be found in a previous study [21]. The reproducibility value as an estimate of the measurement errors for the repeat measurements between two sessions $(n=6)$ was low for the arteriograph $\left(0.18 \mathrm{~m} \cdot \mathrm{s}^{-1}\right)$.

\section{Secondary outcomes}

\section{Anthropometric measurements}

After completing another general information questionnaire, participants were instructed to wear shorts and a T-shirt to the physical exam. They were also required to remove all worn jewelry and metal objects. Once the subjects were barefoot and in their underwear, their body weight $(\mathrm{kg})$ was measured using an electric scale (Model Tanita ${ }^{\circ}$ C $-420^{\circ}$, Tokyo, Japan) with a range of $0-200 \mathrm{~kg}$ and with an accuracy of within $100 \mathrm{~g}$. Height was measured with a portable stadiometer with a precision of $0.1 \mathrm{~cm}$ and a range of $0-2.5 \mathrm{~m}$ (Seca ${ }^{\circ} 274$, Hamburg, Germany). Body mass index (BMI) was calculated as the body weight in kilograms divided by the square of height in meters $\left(\mathrm{kg} / \mathrm{m}^{2}\right)$.

\section{Cardiopulmonary exercise testing and training intensity}

A maximal incremental test was performed by each participant on a treadmill (Precor $\mathrm{TRM}^{\circ} 885$, Italy) using a ramp protocol that simulates field running described by Ramírez-Vélez [18]. The criteria for exercise termination followed the American College of Sports Medicine recommendations [19], identified by an exercise physiologist who was present during each test. Maximum pulmonary oxygen uptake was defined as the mean cardiorespiratory uptake of the last $30 \mathrm{~s}$ of exercise; the maximum HR was registered at the exercise peak.

Although diet was not controlled, participants met with the study's dietician for nutritional assessment and counselling at baseline, and an individualized iso-energetic nutrition intervention plan was developed from the baseline food intake assessment according to participant preferences. This plan was standardized at $50-55 \%$ carbohydrates, $30-35 \%$ total fat, $<7 \%$ saturated fat and $15-22 \%$ protein, distributed across 3-4 meals per day [19].

Physical activity performed outside of the supervised exercise sessions (daily physical activity) was measured using Global Physical Activity Questionnaire for a 10 and 12 weeks [24]. MET-minutes/week were used to estimate the duration and intensity of physical activity during intervention.

\section{Sample size}

The measurement of FMD, validated in several population studies, was selected as the critical variable to calculate the sample size $[25,26]$. We determined the sample size for each group by power calculations using $G^{*}$ Power 3. A randomized clinical trial of the effect of aerobic training on FMD resulted in a standardized effect size (ES) of 0.3 to 0.6 for improvement in endothelial function [27]. An a priori power analysis estimated that a total sample size of 10 participants in each group. It was assumed that FMD would increase by approximately $1 \%$ over 12 weeks.

\section{Statistical analysis}

All of the statistical analyses were performed using SPSS Version 25.0 (Chicago, IL, USA). Data were reported as mean, standard deviation (SD) or standard error (SE). Prior to the planned statistical analyses, a preliminary analysis was conducted (Shapiro-Wilk tests) to confirm the normality of the data. We used a generalized linear model (GLM) with repeated measures to analyze the influence of the different doses of exercise training on components of vascular function outcomes. Cohen's $d$ for effect size (ES) were also calculated to determine the magnitude of the group differences. ESs were classified as small, small-to-medium, and medium-to-large effects $(<0.20,0.2-0.6$ and $0.6-1.2$, respectively) [28].

To classify the participants as "Rs" or "NRs" for improvements in FMD/PWV, the typical error (TE) was calculated, similar to the approach in our recent study $[11,13]$. TE was calculated using the following equation: $\mathrm{TE}=\mathrm{SDdiff} / \sqrt{2}$, where SDdiff is the variance (standard deviation) of the difference in scores observed between the 2 repeats of each test. A "NR" was defined as an individual who failed to demonstrate a decrease or increase (whichever represented a beneficial change) that 
was greater than 2 times the TE. It was assumed that FMD would increase by approximately $0.9 \%$ and PWV reduce $0.5 \mathrm{~m} \cdot \mathrm{s}^{-1}$, over 12 weeks. Chi- squared $\left(\mathrm{X}^{2}\right)$ tests were used to assess the differences between the prevalence of "NRs" post-intervention for each group. All reported $P$ values are two-sided $(P<0.05)$.

\section{Results}

Additional file 1: Figure S1 shows the flowchart of this randomized clinical trial. A total of 28 physical inactive subjects were assessed for eligibility, of which seven were excluded for not meeting the inclusion criteria. Ten participants were randomly allocated to the MCT group, and 11 participants were randomly allocated to the HIT group. After allocation, one participant in the MCT group withdrew for reasons unrelated to this study (i.e., lack of time due to work schedule).

Table 1 presents the within- and between-group differences in vascular parameters following the training program. Peak brachial artery diameter significantly increased in the MCT group (+0.1 [SE 0.1] $\mathrm{mm})$ and HIT group (+0.3 [SE 0.1$] \mathrm{mm})$, with a medium-to-large effect $(d=0.474$ to 0.732$)$, with significant difference between groups: $0.1 \mathrm{~mm}$ (CI $95 \%=$ 0.0 to $0.3 ; P<0.01$ ), indicating positive adaptations following HIT compared with those following MCT. PWV changed by $+0.1 \mathrm{~m} \cdot \mathrm{s}^{-1}$ (SE $\left.0.2, d=0.087\right)$ in the MCT group but decreased by $-0.4 \mathrm{~m} \cdot \mathrm{s}^{-1}$ in the HIT group (SE 0.2, $d=0.497$ ), with significant difference between groups: -0.4 (95\% CI, -0.2 to -0.7$)$. There were no significant treatment effects on other vascular parameters.

Figure $2 \mathrm{a}$ and $\mathrm{b}$ show the mean values for individual changes in FMD (\%) and PWV in both groups. Regarding FMD (\%), the analysis showed a "NR" prevalence of $66 \%$ (6 cases) in the MCT group and 36\% (4 cases) in the HIT group $(p=0.157)$. There was no significant difference in the prevalence of "NRs" for PWV between the MCT and the HIT group (77\% versus $45 \%, p=0.114$ ).

No adverse events were reported over the course of this investigation. All data related to adherence and self-reported physical activity levels are presented in Table 2. Compliance with the study intervention was adequate, with $32 / 36$ (89\%) of participants receiving supervised exercise training. As expected self-reported physical activity increased as a result of training (F [1.65, $135.03]=4.37 ; p<0.001$ ). Pairwise comparison analyses showed that the participants sustained these levels of vigorous or moderate physical activity at the 12-weeks follow-up. Between 10 to 12-week, walking differences over time in both groups was MCT group 945 MET-minutes/week vs HIT group 514 MET-minutes/ week, $(p<0.001)$, but this difference was evident from high-intensity physical activity levels (MCT group 885 MET-minutes/week vs HIT group 1168 MET-minutes/ week, $p<0.001)$.

Table 1 Anthropometric and vascular function parameters at baseline and changes after 12 weeks

\begin{tabular}{|c|c|c|c|c|c|c|c|c|c|}
\hline & \multicolumn{4}{|l|}{ Groups } & \multirow{2}{*}{\multicolumn{2}{|c|}{$\begin{array}{l}\text { Within-Group Change } \\
\text { Mean (SE) }\end{array}$}} & \multirow{3}{*}{$\begin{array}{l}\text { Between-Group } \\
\text { Difference in Change } \\
\text { Mean }(95 \% \mathrm{Cl})\end{array}$} & \multirow{3}{*}{$\begin{array}{l}\text { MCT effect } p \text { value } \\
\text { (Effect size) }\end{array}$} & \multirow{3}{*}{$\begin{array}{l}\text { HIT effect } p \text { value } \\
\text { (Effect size) }\end{array}$} \\
\hline & \multicolumn{2}{|c|}{ Baseline Mean (SD) } & \multicolumn{2}{|c|}{$\begin{array}{l}\text { After } 12 \text { weeks } \\
\text { Mean (SD) }\end{array}$} & & & & & \\
\hline & $\begin{array}{l}\mathrm{MCT} \\
(n=10)\end{array}$ & $\begin{array}{l}\text { HIT } \\
(n=11)\end{array}$ & $\begin{array}{l}\mathrm{MCT} \\
(n=9)\end{array}$ & $\begin{array}{l}\text { HIT } \\
(n=11)\end{array}$ & $\begin{array}{l}\mathrm{MCT} \\
(n=9)\end{array}$ & $\begin{array}{l}\mathrm{HIT} \\
(n=11)\end{array}$ & & & \\
\hline \multicolumn{10}{|l|}{ Anthropometric } \\
\hline Weight, kg & $\begin{array}{l}69.3 \\
(15.3)\end{array}$ & $\begin{array}{l}66.8 \\
(10.9)\end{array}$ & $\begin{array}{l}68.6 \\
(13.5)\end{array}$ & $\begin{array}{l}66.7 \\
(10.5)\end{array}$ & $0.6(0.6)$ & $0.1(0.4)$ & $0.5(-1.2$ to 2.2$)$ & $0.341(0.338)$ & $0.717(0.112)$ \\
\hline $\begin{array}{l}\text { Body mass index, } \\
\mathrm{kg} / \mathrm{m}^{2}\end{array}$ & 23.6 & $25.5(4.2)$ & $23.4(1.2)$ & $24.4(4.2)$ & $0.2(0.2)$ & $1.1(0.9)$ & -0.9 ( -3.3 to 1.4$)$ & $0.393(0.301)$ & $0.274(0.349)$ \\
\hline \multicolumn{10}{|l|}{ Vascular function } \\
\hline$D_{\text {base, }} \mathrm{mm}$ & $2.9(0.5)$ & $2.7(0.4)$ & $3.1(0.4)$ & $3.0(0.4)$ & $0.2(0.1)$ & $0.3(0.1)$ & $-0.1(-0.2$ to 0.1$)$ & $0.278(0.388)$ & $0.043(0.699)$ \\
\hline FMD, \% & $7.1(3.2)$ & $7.2(5.6)$ & $6.1(6.5)$ & $9.1(5.2)$ & $-1.0(2.1)$ & $1.8(1.8)$ & $2.9(-3.0$ to 8.8$)$ & $0.634(0.165)$ & $0.341(0.301)$ \\
\hline$D_{\text {peak }} \mathrm{mm}$ & $3.1(0.6)$ & $2.9(0.4)$ & $3.3(0.5)$ & $3.3(0.5)$ & $0.1(0.1)$ & $0.3(0.1)$ & 0.1 (0.0 to 0.3$)$ & $0.193(0.474)$ & $0.036(0.732)$ \\
\hline$D_{\text {diff }}$ & $0.2(0.1)$ & $0.1(0.1)$ & $0.2(0.2)$ & $0.2(0.1)$ & $0.0(0.0)$ & $0.1(0.0)$ & $0.1(-0.1$ to 0.3$)$ & $0.805(0.085)$ & $0.168(0.449)$ \\
\hline FMDn, \% & $6.5(2.8)$ & $7.4(5.6)$ & $4.9(5.4)$ & $8.1(4.7)$ & $-1.5(1.9)$ & $0.6(1.6)$ & $2.2(-3.2$ to 7.7$)$ & $0.451(0.264)$ & $0.693(0.123)$ \\
\hline $\mathrm{PWV}, \mathrm{m} \cdot \mathrm{s}^{-1}$ & $6.7(0.8)$ & $7.1(1.1)$ & $6.8(0.9)$ & $6.7(1.6)$ & $-0.1(-0.2)$ & $0.4(-0.2)$ & $\begin{array}{l}-0.4(-0.2 \\
\text { to }-0.7)\end{array}$ & $0.800(0.087)$ & $0.130(0.497)$ \\
\hline Alx (aortic), \% & $16.5(5.2)$ & $\begin{array}{l}25.1 \\
(16.5)\end{array}$ & $16.5(8.4)$ & $\begin{array}{l}26.3 \\
(14.6)\end{array}$ & $0.0(2.7)$ & $1.1(2.1)$ & $-1.1(-3.5$ to 1.3$)$ & $0.991(0.004)$ & $0.617(0.156)$ \\
\hline Alx (brachial), \% & $\begin{array}{l}-41.7 \\
(10.4)\end{array}$ & $\begin{array}{l}-24.5 \\
(32.7)\end{array}$ & $\begin{array}{l}-41.7 \\
(16.5)\end{array}$ & $\begin{array}{l}-22.3 \\
(28.9)\end{array}$ & $0.0(5.4)$ & $-2.2(4.2)$ & $-2.2(-6.8$ to 2.4$)$ & $0.998(0.001)$ & $0.619(0.155)$ \\
\hline
\end{tabular}




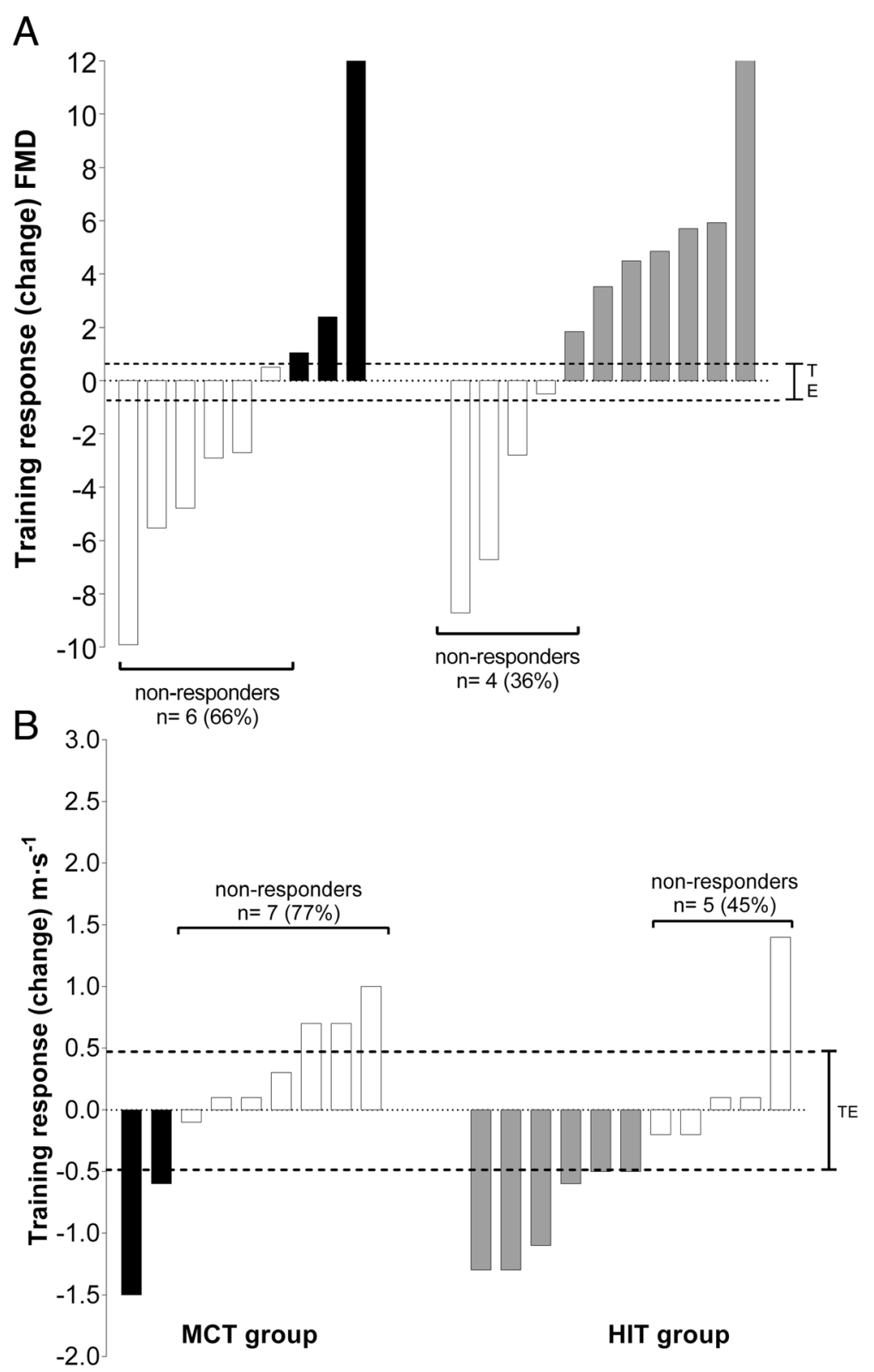

Fig. 2 Differences in the prevalence of non-responders in vascular parameters after 12 weeks training. a FMD $(\%), \mathbf{b}$ PWV $\left(\mathrm{m} \cdot \mathrm{s}^{-1}\right)$

Table 2 Attendance to prescribed exercise sessions and self-reported physical activity

\begin{tabular}{|c|c|c|c|}
\hline Variable & $\mathrm{MCT}(n=9)$ & $\mathrm{HIT}(n=11)$ & $\begin{array}{l}\text { Group effect } \\
\text { (P value) }\end{array}$ \\
\hline Adherence (\% of prescribed sessions completed), mean (SD) & $98.7(3.7)$ & $98.4(2.8)$ & 0.969 \\
\hline Total number of sessions completed, mean (SD) & $32.5(1.3)$ & $32.5(0.9)$ & 0.993 \\
\hline Total MET-minutes/week, mean (SD) & $1100(258)$ & $1031(147)$ & 0.043 \\
\hline \multicolumn{4}{|l|}{ International Physical Activity Questionnaire (10 to 12 week) } \\
\hline Walking MET-minutes/week, mean (SD) & $945(1890)$ & $514(1014)$ & $<0.001$ \\
\hline Moderate MET-minutes/week, mean (SD) & $200(276)$ & $128(260)$ & $<0.001$ \\
\hline Vigorous MET-minutes/week, mean (SD) & $885(712)$ & $1168(588)$ & $<0.001$ \\
\hline
\end{tabular}




\section{Discussion}

To the best of our knowledge, this is the first randomized clinical trial studying the effects of exercise training intensity on vascular parameters and individual responses in physically inactive adults from a Latin-American population. These findings suggest that exercise training induces potent stimuli leading to improvements in vascular parameters (i.e., decrease in arterial wall thickness and increase in endothelial function). Compared to MCT group, HIT is more efficacious for improving FMD and decreasing PWV in physically inactive adults, suggesting the presence of different regulatory mechanisms and time courses for induction.

HIT and MCT on a treadmill have been previously shown to be highly effective in patients with metabolic diseases [29]. Additionally, exercise training has been shown to be an effective therapeutic strategy for vascular function improvement in different clinical populations [30]. A previous meta-analysis showed that HIT is more potent than MIT in enhancing FMD, with a mean difference of 2.2\% [31]. Specifically, this review suggested that a $4 \times 4$ HIT protocol three times per week for at least 12 weeks is an effective form of exercise for enhancing vascular functions. Our study showed a mean difference of $2.9 \%$ in FMD between groups; however, the difference was not significant. Along the same line, our data indicate that while brachial artery diameter increased as a result of exercise, arterial function assessed by PWV $\left(\mathrm{m} \cdot \mathrm{s}^{-1}\right)$ decreased at high levels of exercise (Table 1$)$.

It is conceivable that substantial and/or sustained increases in shear forces that occur during exercise bouts may be associated with improving FMD, because stimulation of vasodilation post-exercise may result in the inhibition of related biochemical pathways [30]. In line with this, a meta-analysis of prospective studies reported a $13 \%$ reduction in the risk of cardiovascular events with a $1 \%$ increase in FMD; therefore, the magnitude of change in FMD following HIT (pre- vs. post-HIT + $1.8 \%$ ) was deemed to be clinically significant in our study $(d=0.301$ [small-to-medium effect size] $)$.

Differences in exercise and experimental protocols in our study may have also contributed to discrepancies in our findings; however, this hypothesis remains to be tested. Our study showed that exercise intensity influences FMD response; however, FMD following exercise was attenuated in the MCT group but enhanced in the HIT group. Siasos et al. [7] suggested that both acute HIT and MCT can favorably affect endothelial function in healthy young adults, indicating another cardioprotective effect of exercise preventing the progression of atherosclerosis. The effects of these intense exercise regimens on FMD reflect a combination of hemodynamic changes and endothelial nitric oxide-dependent mechanisms [4, 10]. Exercise induces increases in blood flow, and augmented blood flow causes vasodilation, which directly impacts the magnitude of FMD [22, 32].

Regarding arterial wall parameters, aerobic exercise seems to significantly improve arterial stiffness, and this effect is enhanced at higher intensities of aerobic exercise and in participants with greater baseline arterial stiffness [31, 33]. PWV is widely recognized as a direct marker of arterial stiffness ${ }^{33}$. AIx index (aortic and brachial), are being more frequently used in studies as parameters of wave reflection [34]. In addition, an increase in PWV is linked with increased rates of cardiovascular incidences related to increased left ventricular afterload and wasted left ventricular energy [31, 33]. In this study, PWV increase by $+0.1 \mathrm{~m} \cdot \mathrm{s}^{-1}$ (SE $0.2, d=0.087$ ) in the MCT group, but decreased by $-0.4 \mathrm{~m} \cdot \mathrm{s}^{-1}$ in the HIT group (SE 0.2, $d=0.497$ ), with significant difference between groups: -0.4 (95\% CI, -0.2 to -0.7$)$. A previous systematic review and meta-analysis of RCTs reported that every $1 \mathrm{~m} \cdot \mathrm{s}^{-1}$ increase in PWV is associated with a $12-14 \%$ increase in the risk of cardiovascular events and a $13-15 \%$ increase in the risk of CVD mortality. On the other hand, it was reported that aerobic exercise reduced PWV by $0.63 \mathrm{~m} \cdot \mathrm{s}^{-1}$, which may be translated into an $8 \%$ reduction in cardiovascular events and a $9 \%$ reduction in cardiovascular mortality. Furthermore, subgroup analyses suggested that there may be bigger effects on PWV and, consequently, on cardiovascular events and mortality of aerobic exercise in higher risk participants (with $\mathrm{PWV} \geq 8 \mathrm{~m} / \mathrm{s}$ at baseline) and with longer durations of aerobic exercise (> 10 weeks) $[31,33]$. In this line, HIT protocols seems to have a greater effect on peripheral than on central indices of arterial stiffness [7], which could justify our findings. However, several discrepancies between findings could also be due to differences in exercise modes or durations of HIT intervals; Ramos et al. [8] and Sawyer et al. [15] suggest that metabolic responses to HIT vary depending on the duration of the work-rest intervals.

Evidence from systematic reviews and experimental studies has demonstrated a positive effects of various exercise modalities (aerobic, resistance and combined training) on endothelial functions $[7,29,31]$, but there are controversies regarding the effects of HIT on indices pertaining to arterial stiffness and wave reflection [7, 31, 35]. The mechanism by which HIT significantly reduces PWV more than MCT does could be associated with reduced exposure of the vasculature to reactive oxygen species that are often observed during high-volume exercise [36]. It is also possible that the higher volume of exercise in the HIT group may have resulted in the requirement of longer time for PWV recovery from repeated HIT bouts, thereby providing a more accurate representation of the cumulative effect of exercise intervention. These results may help identify the vascular wall that is more responsive and, 
conversely, the wall that is more resistant to the arterial stiffness-lowering effects of HIT [35].

On the order hand, the phenomenon of "NR" has been explored on performance variables [1] using MCT [12], resistance training [37], or HIT [11] in different age groups such as children [13], adults [29], and older populations [38]. Regarding FMD (individual responses), our analysis showed an "NR" rate of $66 \%$ in the MCT group and $36 \%$ in the HIT group $(P=0.157)$. Regarding PWV $\left(\mathrm{m} \cdot \mathrm{s}^{-1}\right)$, an analysis showed that the prevalence of no-responder was 77\% (7 cases) in the MCT group and $45 \%$ (5 cases) in the HIT group $(P=0.114)$. This information can be useful when there are more than one risk factor to improve in physically inactive populations, and this knowledge can be useful for choosing exercise interventions with low rates of "NR" and high rates of improvements in particular outcomes. The data from some studies support our conclusion that exercise intensity plays an important role in modulating adaptations in vascular functions in response to exercise [4, 31, 32]. In line with this, several previous studies have reported increases $[39,40]$ decreases [27] or lack of change [10] in FMD following different exercise protocols. Unfortunately, none of these studies on exercise interventions reported on the rate of "NR". Although some misleading studies have claimed the lack of non-responders in 4-week training intervals ${ }^{1}$, more recently, this phenomenon has been confirmed after 6 weeks and 6-8-months of exercise by relevant authors in the field [1].

In any case, the term "NR" may be related more to semantics, as the authors demonstrate a lack of response in some of the chosen outcomes (e.g., $\mathrm{VO}_{2}$ peak, lean body mass, muscle strength, health status, etc.) across participants. Even the authors of reports that refute the so-called 'myth' of exercise non-response might agree that the term "NR" depends solely on the chosen clinical outcomes and that a non-responder for one outcome may not be a non-responder in another outcome $[1,11]$. As technology advances and our understanding of the mechanisms driving exercise responses improves, scientists can continue to narrow the focus on clinical outcomes that are critical for improving the health of an individual, and healthcare practitioners can thus recommend exercise regimens on an individual basis rather than broadly suggesting the same exercise regimens for everyone.

Regardless of the mechanisms, it has been suggested that HIT may impair endothelium-dependent vasodilation due to an increase in reactive oxygen species, resulting in a reduction in nitric oxide bioavailability. Additionally, responses in FMD are inversely proportional to baseline arterial diameter. Further studies are necessary to establish optimal exercise training interventions for improving vascular health assessed by measuring FMD. Additionally, differences between the effects of different exercise regimens could be due to variability in their ability to generate greater blood flow through vessels supplying oxygen to the working muscles, which could in turn promote greater shear stress-induced nitric oxide bioavailability [39] and induce favorable endothelial adaptations [40]. In this context, several biologically plausible mechanisms may be used to explain the effects of exercise on the modulation of endothelial functions and arterial stiffness. It is widely known that exercise has the potential to reduce oxidative stress by increasing the efficiency of the antioxidant system, eventually improving endothelial dysfunction [40]. The main physiological mechanisms involve the up-regulation of endothelial nitric oxide synthase activity, as demonstrated in cell culture, animal and human studies, with a subsequent reduction in the expression of nicotinamide adenine dinucleotide (phosphate) (NAD $(\mathrm{P}) \mathrm{H})$-dependent oxidase and the stimulation of free radical-scavenging systems that affect the levels of copper/zinc-containing superoxide dismutase, extracellular superoxide dismutase, glutathione peroxidase and glutathione [37]. Other studies have examined indices of antioxidant capacity, oxidative stress, and nitric oxide bioavailability as possible sources of improved FMD or reduced PWV. Future examinations should consider assessing these indices to fill in the gaps in the literature in their specific population of interest.

We did not observe improvements in AIx (\% aortic or $\%$ brachial) body weight, or BMI, with exercise training, and therefore not likely clinically relevant. However, these findings should not deter future investigations from examining these indices. It remains possible that a larger sample size, or a sample with more clinically relevant pre-training states, such as hypertension, obesity, and elevated PWV, may experience more dramatic reductions in these indices with MCT or HIT training.

The strengths of this study include the use of state-of-the-art measures of vascular functions with supervised exercise training in a non-clinical setting. In addition, adherence to the intervention was $\approx 89 \%$. All subjects completed 32 of 36 exercise sessions, and research technicians supervised each session while HR was being monitored. A primary limitation of this study was the lack of a true control group without exercise. Thus, we are unable to determine causality in our interpretation of the observed exercise-induced improvements in cardiovascular health parameters. Furthermore, in studies comparing HIT and MCT that have included a control group (no-exercise), no changes in FMD were observed in the control group [29]. Due to this and other limitations (e.g., single site design), it is important to not over-interpret the results of this RCT [17]. Other limitations of this study include the lack of control over tobacco usage. Additionally, indices other than post-occlusion reactive hyperemia flux were not assessed in the present 
study. However, we cannot determine the directions of the associations nor any causality observed in this study with absolute certainty. Lastly, we did not measure plasma nitric oxide, anti-oxidants and cytokines; however, any additional information provided by these measures may help to explain our findings.

Identifying the training regimen that has the most beneficial effects on each parameter could potentially lead to enhanced precision in prescribing exercise training intensity to achieve optimal outcomes in this population. Under the conditions of the present study, physically inactive adults in both groups experienced changed in FMD. Not all vascular function measured responded the same to this type of exercise, suggesting different regulatory mechanisms and time courses for induction.

\section{Conclusion}

This study demonstrates the efficacy of HIT in enhancing the cardioprotective effects of exercise on the progression of atherosclerosis in a physically inactive population. However, compared to MCT group, HIT is more efficacious for improving FMD and decreasing PWV, in physically inactive adults. Identifying the mechanisms of adaptation may help to optimize the exercise program to target these mechanisms.

\section{Additional file}

Additional file 1: Figure S1. CONSORT guidelines flow diagram for enrolment and randomisation HIT-Heart Study. (TIF 265 kb)

\section{Abbreviations \\ AHA: American Heart Association; CEMA: Centre of studies in physical activity measurements (in Spanish); CVD: Cardiovascular disease; FMD: Flow- mediated vasodilation; HIT: High Intensity interval training; HRR: Heart rate reserve; ICC: Intra-class correlation coefficients; MCT: Moderate intensity training; METs: Units of metabolic equivalence; NRs: Non-responders; PA: Physical activity; PWV: Aortic pulse wave velocity; Rs: Responders}

\section{Acknowledgements}

We would like to thank and acknowledge the enthusiastic group of test participants who made this study possible.

\section{Funding}

This study was part of the project entitled "Body Adiposity Index and Biomarkers of Endothelial and Cardiovascular Health in Adults: Effect of Physical Training", which was funded by Centre for Studies on Measurement of Physical Activity, School of Medicine and Health Sciences, Universidad del Rosario (Code No FIUR DN-BG001). The funder had no role in the study design, data collection, data analysis and interpretation, preparation of the manuscript, or decision to publish.

\section{Availability of data and materials}

The datasets used and/or analyzed during the present study are available from the corresponding author on reasonable request.

\section{Authors' contributions}

RR-V conceived and designed the project. RR-V, JEC-B, RG-G and PAH-Q reviewed the literature studies and conducted data extraction. RR-V and JEC$B$ conducted data analyses. RR-V, RG-G and MI were responsible for data interpretation. AT-S, MI and RR-V drafted the manuscript, and PAH-Q, and MI revised it critically for intellectual contributions. MI and RR-V coordinate the study development. All authors reviewed and edited the manuscript. All authors read and approved the final manuscript.

\section{Ethics approval and consent to participate}

Details of the study design and methods of the primary HIT-Heart Study trial have been described elsewhere (ClinicalTrials.gov ID: NCT02738385; April 14th, 2016). The study was performed in accordance with the Declaration of Helsinki (2000) and was approved by the local office for Medical Research Ethics Committee of The University of Santo Tomás, Colombia (ID 27-05002015). All participants provided written informed consent before participating in the study.

\section{Consent for publication}

Not applicable.

\section{Competing interests}

The authors declare that they have no competing interests.

\section{Publisher's Note}

Springer Nature remains neutral with regard to jurisdictional claims in published maps and institutional affiliations.

\section{Author details}

${ }^{1}$ Centro de Estudios para la Medición de la Actividad Física "CEMA". Escuela de Medicina y Ciencias de la Salud, Universidad del Rosario, Bogotá, DC, Colombia. ${ }^{2}$ Grupo GICAEDS. Programa de Cultura Física, Deporte y Recreación, Universidad Santo Tomás, Bogotá, DC, Colombia. ${ }^{3}$ Department of Physical Activity Sciences, Universidad de Los Lagos, Osorno, Chile. ${ }^{4}$ Research Nucleus in Health, Physical Activity and Sports, Universidad de Los Lagos, Osorno, Chile. ${ }^{5}$ Department of Health Sciences, Public University of Navarre, CIBER de Fragilidad y Envejecimiento Saludable (CB16/10/00315), Tudela, Navarre, Spain. 'Laboratorio de Ciencias de la Actividad Física, el Deporte y la Salud, Universidad de Santiago de Chile, USACH, Santiago, Chile. ${ }^{7}$ Martinos Center for Biomedical Imaging, Department of Radiology, Massachusetts General Hospital, Harvard Medical School, Boston, MA, USA. ${ }^{8}$ Neurovascular Science Group, Fundación Cardiovascular de Colombia, Floridablanca, Santander, Colombia. ${ }^{9}$ Connors Center for Women's Health and Gender Biology, Brigham and Women's Hospital, Harvard Medical School, Boston, MA, USA.

Received: 12 June 2018 Accepted: 21 January 2019

Published online: 04 February 2019

References

1. Bouchard C, Blair SN, Katzmarzyk PT. Less sitting, more physical activity, or higher fitness? Mayo Clin Proc. 2015;90:1533-40.

2. Kohl HW 3rd, Craig CL, Lambert EV, Inoue S, Alkandari JR, Leetongin G, Kahlmeier S. Lancet physical activity series working group. The pandemic of physical inactivity: global action for public health. Lancet. 2012;380:294-305.

3. Joyner MJ, Green DJ. Exercise protects the cardiovascular system: effects beyond traditional risk factors. J Physiol. 2009;587:5551-8.

4. Green DJ, Hopman MT, Padilla J, Laughlin MH, Thijssen DH. Vascular adaptation to exercise in humans: role of hemodynamic stimuli. Physiol Rev. 2017:97:495-528.

5. Ramos JS, Dalleck LC, Tjonna AE, Beetham KS, Coombes JS. The impact of high-intensity interval training versus moderate-intensity continuous training on vascular function: a systematic review and meta-analysis. Sports Med. 2015;45:679-92.

6. Schjerve IE, Tyldum GA, Tjønna AE, Stølen T, Loennechen JP, Hansen HE, Haram PM, Heinrich G, Bye A, Najjar SM. Both aerobic endurance and strength training programmes improve cardiovascular health in obese adults. Clin Sci. 2008;115:283-93.

7. Siasos G, Athanasiou D, Terzis G, Stasinaki A, Oikonomou E, Tsitkanou S, Kolokytha T, Spengos K, Papavassiliou AG, Tousoulis D. Acute effects of different types of aerobic exercise on endothelial function and arterial stiffness. Eur J Prev Cardiol. 2016;23:1565-72.

8. Ramos JS, Dalleck LC, Ramos MV, Borrani F, Roberts L, Gomersall S, Beetham KS, Dias KA, Keating SE, Fassett RG, Sharman JE, Coombes JS. 12 min/week of high-intensity interval training reduces aortic reservoir pressure in 
individuals with metabolic syndrome: a randomized trial. J Hypertens. 2016; 34:1977-87.

9. Weber T, Beijer Å, Rosenberger A, Mulder E, Yang P, Schönau E, Bloch W, Rittweger J. Vascular adaptations induced by 6 weeks WBV resistance exercise training. Clin Physiol Funct Imaging. 2013;33:92-100.

10. Green D, Cheetham C, Mavaddat L, Watts K, Best M, Taylor R, O'Driscoll G. Effect of lower limb exercise on forearm vascular function: contribution of nitric oxide. Am J Physiol Heart Circ Physiol. 2002;283:H899-907.

11. Álvarez C, Ramírez-Campillo R, Ramírez-Vélez R, Izquierdo M. Effects and prevalence of nonresponders after 12 weeks of high-intensity interval or resistance training in women with insulin resistance: a randomized trial. J Appl Physiol (1985). 2017;122:985-96.

12. Bonafiglia JT, Rotundo MP, Whittall JP, Scribbans TD, Graham RB, Gurd BJ. Inter-individual variability in the adaptive responses to endurance and Sprint interval training: a randomized crossover study. PLoS One. 2016; 11(12):e0167790.

13. Álvarez C, Ramírez-Campillo R, Ramírez-Vélez R, Izquierdo M. Effects of 6weeks high-intensity interval training in schoolchildren with insulin resistance: influence of biological maturation on metabolic, body composition, cardiovascular and performance non-responses. Front Physiol. 2017:8:444.

14. Mann TN, Lamberts RP, Lambert MI. High responders and low responders: factors associated with individual variation in response to standardized training. Sports Med. 2014;44:1113-24.

15. Sawyer BJ, Tucker WJ, Bhammar DM, Ryder JR, Sweazea KL, Gaesser GA. Effects of high-intensity interval training and moderate-intensity continuous training on endothelial function and cardiometabolic risk markers in obese adults. J Appl Physiol. 2016;121:279-88.

16. Ramírez-Vélez R, Lobelo F, Izquierdo M. Exercise for disease prevention and management: a precision medicine approach. J Am Med Dir Assoc. 2017;18:633-4.

17. Ramírez-Vélez R, Tordecilla-Sanders A, Téllez-T LA, Camelo-Prieto D, Hernández-Quiñonez PA, Correa-Bautista JE, Garcia-Hermoso A, RamirezCampillo R, Izquierdo M. Similar cardiometabolic effects of high- and moderate-intensity training among apparently healthy inactive adults: a randomized clinical trial. J Transl Med. 2017;15:118.

18. Ramírez-Vélez R, Tordecilla-Sanders A, Téllez-T LA, Camelo-Prieto D, Hernández-Quiñonez PA, Correa-Bautista JE, Garcia-Hermoso A, RamírezCampillo R, Izquierdo M. Effect of moderate versus high-intensity interval exercise training on heart rate variability parameters in inactive LatinAmerican adults: a randomised clinical trial. J Strength Cond Res. 2017. https://doi.org/10.1519/JSC.0000000000001833 Epub ahead of print.

19. American College of Sports Medicine. Position stand: the recommended quantity and quality of exercise for developing and maintaining cardiorespiratory and muscular fitness, and flexibility in healthy adults. Med Sci Sports Exerc. 1998;30:975-91.

20. Lloyd-Jones DM, Hong Y, Labarthe D, American Heart Association Strategic Planning Task Force And Statistics Committee. Defining and setting national goals for cardiovascular health promotion and disease reduction: the American Heart Association's strategic impact goal through 2020 and beyond. Circulation. 2010;121:586-613.

21. Ramírez-Vélez R, Correa-Rodríguez M, Tordecilla-Sanders A, Aya-Aldana V, Izquierdo M, Correa-Bautista JE, Álvarez C, Garcia-Hermoso A. Exercise and postprandial lipemia: effects on vascular health in inactive adults. Lipids Health Dis. 2018;17(1):69.

22. Atkinson G. Shear rate normalization is not essential for removing the dependency of flow-mediated dilation on baseline artery diameter: past research revisited. Physiol Meas. 2014;35:1825-35.

23. Ring $M$, Eriksson MJ, Zierath JR, Caidahl K. Arterial stiffness estimation in healthy subjects: a validation of oscillometric (Arteriograph) and tonometric (SphygmoCor) techniques. Hypertens Res. 2014;37:999-1007.

24. Hoos T, Espinoza N, Marshall S, Arredondo EM. Validity of the global physical activity questionnaire (GPAQ) in adult Latinas. J Phys Act Health. 2012;9:698-705.

25. Hashimoto M, Kozaki K, Eto M, Akishita M, Ako J, lijima K, Kim S, Toba K, Yoshizumi M, Ouchi Y. Association of coronary risk factors and endotheliumdependent flow-mediated dilatation of the brachial artery. Hypertens Res. 2000:23:233-8

26. Hashimoto M, Akishita M, Eto M, Kozaki K, Ako J, Sugimoto N, Yoshizumi M, Toba $K$, Ouchi $Y$. The impairment of flow-mediated vasodilatation in obese men with visceral fat accumulation. Int J Obes Relat Metab Disord. 1998;22:477-84.
27. Early KS, Stewart A, Johannsen N, Lavie CJ, Thomas JR, Welsch M. The effects of exercise training on brachial artery flow-mediated dilation: a meta-analysis. J Cardiopulm Rehabil Prev. 2017;37:77-89.

28. McGough JJ, Faraone SV. Estimating the size of treatment effects: moving beyond P values. Psychiatry (Edgmont). 2009;6:21-9.

29. Tjønna AE, Lee SJ, Rognmo Ø, Stølen TO, Bye A, Haram PM, Loennechen JP, Al-Share QY, Skogvoll E, Slørdahl SA, Kemi OJ, Najjar SM, Wisløff U. Aerobic interval training versus continuous moderate exercise as a treatment for the metabolic syndrome: a pilot study. Circulation. 2008;118:346-54.

30. Black MA, Green DJ, Cable NT. Exercise prevents age-related decline in nitric-oxide-mediated vasodilator function in cutaneous microvessels. J Physiol. 2008;586:3511-24.

31. Ashor AW, Lara J, Siervo M, Celis-Morales C, Oggioni C, Jakovljevic DG, Mathers JC. Exercise modalities and endothelial function: a systematic review and dose-response meta-analysis of randomized controlled trials. Sports Med. 2015;45:279-96.

32. Pyke KE, Tschakovsky ME. The relationship between shear stress and flowmediated dilatation: implications for the assessment of endothelial function. J Physiol. 2005;568:357-69.

33. Ashor AW, Lara J, Siervo M, Celis-Morales C, Mathers JC. Effects of exercise modalities on arterial stiffness and wave reflection: a systematic review and meta-analysis of randomized controlled trials. PLoS One. 2014;9(10):e110034.

34. Roman MJ, Devereux RB, Kizer JR, Okin PM, Lee ET, Wang W, Umans JG, Calhoun D, Howard BV. High central pulse pressure is independently associated with adverse cardiovascular outcome the strong heart study. J Am Coll Cardiol. 2009:54:1730-4.

35. Mora-Rodriguez R, Ramirez-Jimenez M, Fernandez-Elias VE, Guio de Prada MV, Morales-Palomo F, Pallares JG, Nelson RK, Ortega JF. Effects of aerobic interval training on arterial stiffness and microvascular function in patients with metabolic syndrome. J Clin Hypertens (Greenwich). 2017. https://doi. org/10.1111/jch.13130 Epub ahead of print.

36. Maclnnis MJ, Gibala MJ. Physiological adaptations to interval training and the role of exercise intensity. J Physiol. 2017;595:2915-30.

37. Davidsen PK, Gallagher IJ, Hartman JW, Tarnopolsky MA, Dela F, Helge JW. High responders to resistance exercise training demonstrate differential regulation of skeletal muscle microRNA expression. J Appl Physiol. 2011;110:309-17.

38. Chmelo EA, Crotts Cl, Newman JC, Brinkley TE, Lyles MF, Leng X, Marsh AP, Nicklas BJ. Heterogeneity of physical function responses to exercise training in older adults. J Am Geriatr Soc. 2015;63:462-9.

39. Harris RA, Padilla J, Hanlon KP, Rink LD, Wallace JP. The flow-mediated dilation response to acute exercise in overweight active and inactive men. Obesity (Silver Spring). 2008;16:578-84.

40. Johnson BD, Padilla J, Wallace JP. The exercise dose affects oxidative stress and brachial artery $\mathrm{fl} \mathrm{ow}$-mediated dilation in trained men. Eur J Appl Physiol. 2012;112:33-42.

\section{Ready to submit your research? Choose BMC and benefit from:}

- fast, convenient online submission

- thorough peer review by experienced researchers in your field

- rapid publication on acceptance

- support for research data, including large and complex data types

- gold Open Access which fosters wider collaboration and increased citations

- maximum visibility for your research: over $100 \mathrm{M}$ website views per year

At $\mathrm{BMC}$, research is always in progress.

Learn more biomedcentral.com/submission 